\title{
The importance of soil archives for microbial ecology
}

\section{Jan Dolfing and Youzhi Feng}

In a recent Comment article (The importance of sample archiving in microbial ecology. Nature Rev. Microbiol. 12, 789-790 $(2014))^{1}$, Cary and Fierer asserted that, as a research community, we must "develop robust strategies for long-term storage and archiving of samples in order to fully develop, and protect, the scientific record" and "where possible, make them available to other researchers" (REF. 1). Indeed, these are important issues in microbial ecology; here, we wish to extend the observations of Cary and Fierer by highlighting the existence of soil archives and briefly discussing their value to microbial ecologists ${ }^{2}$.

Institutions in several countries systematically curate collections of archived soil samples (TABLE 1), which are generally obtained from large systematic surveys and long-term field experiments. These samples have been archived because of their immanent value to many diverse research fields, including microbial ecology. As Cary and Fierer posit, the analytical techniques available for data generation tend to become more sensitive over time, and to answer future research questions unforeseen at the time of sampling, the preservation of these samples for subsequent studies is crucial. The classic soil collections, such as those in Rothamsted and Wageningen (established in 1846 and 1879, respectively), were initiated by chemists working with soill, and used only air drying and sieving as preservation treatments. With the advent of modern molecular techniques, it is now possible for microbiologists to carry out higher-resolution analyses on these samples to determine their microbial and genetic compositions $^{2-4}$. For example, qPCR-based molecular techniques have been used to quantify the presence of antibiotic resistance genes over time in historical soil samples from the Netherlands $s^{5}$. These data have shown that the presence of antibiotic resistance genes in agricultural soils has substantially increased since 1940, concomitant with the increase in industrial antibiotic production. In another study, a high prevalence of Bacillus asahii (which is thought to play a key part in soil fertility through its ability to accelerate carbon and phosphorous cycling) was observed in archived and fresh soil samples containing organic manure, whereas the organism was essentially absent in soils fertilized with inorganic

\begin{tabular}{ll}
\hline $\begin{array}{l}\text { Table } 1 \text { | Examples of soil archives containing sieved soil samples } \\
\text { Country }\end{array}$ & Curator \\
\hline China & Institute of Soil Science, Chinese Academy of Sciences \\
\hline Denmark & Danish Institute of Agricultural Sciences, Foulum \\
\hline France & Institut National de la Recherche Agronomique, Versailles \\
\hline India & Punjab Agricultural University \\
\hline The Netherlands & Alterra, Wageningen \\
\hline United Kingdom & Rothamsted Experimental Station \\
\hline United States & Agricultural Research Service, US Department of Agriculture \\
\hline United States & Duke University \\
\hline
\end{tabular}

fertilizer. The availability of archived soil samples made it possible to trace back the origin of the bacterium and revealed that the indigenous $B$. asahii population in the soil took 2-4 years to become dominant ${ }^{6}$. In the latter example, B. asahii was also isolated and cultivated from the archived samples. Importantly, one potential caveat with archived soils is that air drying as a preservation technique is known to disrupt bacterial and eukaryotic diversity within the sample ${ }^{4}$, so caution is warranted in quantitative studies that examine microbial abundance in samples preserved in this manner.

The studies described above illustrate the usefulness of existing soil archives in providing a readily available source of ecological information that is relevant to microbial ecology, probably more than we can currently fathom. We reinforce the observation of Cary and Fierer that exceedingly valuable information that was not sought at the time of sampling can be retrieved from archived samples at a later date, and we encourage the use of archiving for samples other than soil to maximize the research potential of such samples.

Jan Dolfing is at the School of Civil Engineering and Geosciences, Newcastle University, Newcastle NE1 7RU, UK.

Youzhi Feng is at the State Key Laboratory of Soil and Sustainable Agriculture, Institute of Soil Science, Chinese Academy of Sciences, Nanjing 210008, China.

Correspondence to J.D. e-mail: jan.dolfing@ncl.ac.uk doi: 10.1038/nrmicro3382-c1

1. Cary, S. C. $\&$ Fierer, N. The importance of sample archiving in microbial ecology. Nature Rev. Microbiol. 12, 789-790 (2014).

2. Dolfing, J. et al. Microbial diversity in archived soils. Science 306, 813 (2004).

3. Clark, I. M. \& Hirsch, P. R. Survival of bacterial DNA and culturable bacteria in archived soils from the Rothamsted Broadbalk experiment. Soil Biol. Biochem. 40, 1090-1102 (2008).

4. Tzeneva, V. A. et al. Effect of soil sample preservation, compared to the effect of other environmental variables, on bacterial and eukaryotic diversity. Res. Microbiol. 160, 89-98 (2009).

5. Knapp, C. W., Dolfing, J., Ehlert, P. A. \& Graham, D. W. Evidence of increasing antibiotic resistance gene abundances in archived soils since 1940. Environ. Sci. Technol. 44, 580-587 (2010).

6. Feng, Y. Z. et al. Bacillus asahii comes to the fore in organic manure fertilized alkaline soils. Soil Biol. Biochem. 81, 186-194 (2015).

Competing interests statement The authors declare no competing interests. 